\title{
Influence of an Exit Wall Boundary on the Flow of a Circular Jet
}

\author{
A.P. $\operatorname{Vouros}^{1 \dagger}$, M.D. Mentzos ${ }^{2}$, E.I. Xanthopoulos ${ }^{1}$, A.E. Filios ${ }^{1}$ and D.P. Margaris ${ }^{2}$ \\ ${ }^{1}$ Laboratory of Fluid Mechanics and Turbomachinery, School of Pedagogical and Technological Education \\ (ASPETE), Heraklion, Athens, GR-14121, Greece \\ ${ }^{2}$ Fluid Mechanics Laboratory and Applications, Department of Mechanical Engineering and Aeronautics, University \\ of Patras, Patras, GR-26500, Greece \\ †Corresponding Author Email: avouros@meed-aspete.net
}

(Received March 12, 2012; accepted July 21, 2012)

\begin{abstract}
The flow of the axisymmetric jet constitutes a subject of research from the origins of fluid dynamics; however it remains a subject of interest due to the recent findings that denote the influence of flow and geometry conditions in configurations that diverge from the theoretical "free-jet" case. In the present study, the effect of a wall boundary produced by a circular disk of twice the jet diameter, which is imposed on the exit of the jet is investigated experimentally and numerically. Computational simulations are performed to predict the flow characteristics incorporating different turbulence models (k- $\varepsilon$ and Reynolds Stress) and solvers (Simplec and Coupled). Supportive pressure measurements are used to evaluate the predictions within the initial region of a circular jet at two Reynolds numbers $(50,000$ and 65,000$)$. The results indicate that the presence of the exit wall boundary results to the formation of recirculation zone behind the exit, which prevents the entrainment of ambient fluid. Comparing with the flow field of the free from confinement jet, it is shown that the imposition of the wall has a minor effect on the mean velocity field; it is however capable of altering the turbulent flow properties, including the normal and the Reynolds shear stresses, in the region before the establishment of the self-similarity zone.
\end{abstract}

Keywords: Axisymmetric jet flow, Turbulence, Computational fluid dynamics, Measurements

\section{INTRODUCTION}

The axisymmetric jet flow constitutes an illustrative example in the study of fluid dynamics and turbulence while it is utilized in numerous technical applications including mixing, cooling, combustion etc. The most classical studies of the literature include those of Wygnaski and Fielder (1967), Panchapakesan and Lumley (1993) and Hussein et al. (1994). Those studies revealed the differences in the mean and turbulent structure of jets that emanate at different Reynolds numbers in different spatial spaces (else noted as enclosures). George (1989) discussed the role of the flow and geometry conditions, concluding that they can substantially affect the flow during its development and also the far-field characteristics. In the far-field, that was characterized the region after 60 or 70 diameters from the jet exit, self-similar profiles could be produced when velocity and radial extent were used in nondimensional form using the local velocity maximum and the jet half-width (i.e. the radial position where velocity attains the half of its centerline value) in each axial distance. Even if this form however, the results produced by different experiments or computational efforts did not seem to be "universal" so that during the last decades research has been devoted to the identification of the possible effects arising from the different initial conditions imposed at configurations that diverge from the theoretical "free"-jet flow.

A large number of studies have been focused on the influence of the Reynolds number of the jet. Weisgrabber and Liepmann (1998) presented measurements of a water jet in the region before the establishment of the self-similar profiles using Reynolds number 5000 and 16000. Borg et al. (2001) and Cowen et al. (2001) recorded jets issuing at Reynolds number 6000 and 4000, respectively. Kwon and Seo (2005), Xia and Lam (2009) and Todde et al. (2009) studied in particular the influence of low Reynolds numbers reaching values that lead to transitional or even laminar jets. The common outcome of all the above studies was that the increase of the Reynolds number results to a wider spreading of the jet and also, to an increase of the magnitudes of the turbulent average statistics.

The effect of the geometrical shape at the exit of the jet was also discussed in several studies. Antonia and Zhao (2001), Xu and Antonia (2002), and Quinn (2006) presented measurements conducted in the velocity field of jets issuing from smoothed contraction, straight pipe 
and sharped-edged orifices concluding that straight pipe jets form narrower profiles of the turbulent statistics with lower maximum values at the same axial distances downstream from the jet exit. On the other hand, based on the half-width and the turbulent intensities, it was found that the sharp-edged geometry at the exit can produce more efficient mixing with the ambient resulting to a wider extent of the flow.

Fewer studies presented the effect of confinements imposed peripherally at the jet flow. A parametric computational study was presented by Kandakure et al. (2008), who focused on the effect of the enclosure size around a round jet that evolve inside a cylinder while Borean et al. (1998) discussed the influence of the confinement combined with a co-flowing stream inside a cylindrical pipe. Both these studies mentioned the strong degradation of the entrainment process, which results to narrower radial extent of the jet and also the lower values of the turbulent statistics compared to those of typical "free" jets.

To the authors' knowledge, a single study refers to the influence of a flat plate imposed as a wall boundary on the exit of an axisymmetric jet (Abdel-Rahman et al. 1997). Abdel-Rahman (2010) recently reviewed these measurements - the only available data relating round jets - along with the data of Alnahhal et al. (2011), who studied a similar influence but in a plane turbulent jet. Although he mentioned that the use of endplates appears to restrict the mixing with ambient air providing a slower spreading rate than that of free from confinements jets, he also admitted that more systematic studies are necessary in order to clarify the behavior of the flow under this particular influence.

The lack of literature regarding the use of exit wall boundaries (else denoted as endplates) in axisymmetric jets strongly motivated the work presented here. In the present study, Reynolds averaging computational models are incorporated as an introductory approach in the study of the flow with and without this influence. The effect of the imposition of a circular disk placed at the exit of the jet is investigated for two Reynolds numbers of the same order of magnitude, namely 50,000 and 65,000. Results include statistical averages of the velocity field including the mean axial and radial velocity components, the axial and radial normal stresses and the corresponding Reynolds shear stresses. Supportive Prandtl tube measurements are presented at axial distances that refer to the region of the initial growth of jet flow while predictions are presented for a larger region, up to 25 diameters downstream of the jet exit. The study has two main objectives. The first is to examine the effectiveness of low cost computational models in order to reveal the flow characteristics according to the different initial conditions while the second is to offer new results and discuss the arising trends in comparison to earlier data of the relevant literature.

\section{Methodology}

\subsection{Experimental Facility}

A variable speed axial flow fan produces the air flow in a cylindrical duct of $298 \mathrm{~mm}$ diameter and $653 \mathrm{~mm}$ length. Two contractions in tandem array accelerate smoothly the flow to the duct exit, where the jet is produced. The exit of the downstream contraction has diameter $50 \mathrm{~mm}$ and it suits a circular disk placed perpendicular to the centerline with a diameter $100 \mathrm{~mm}$.

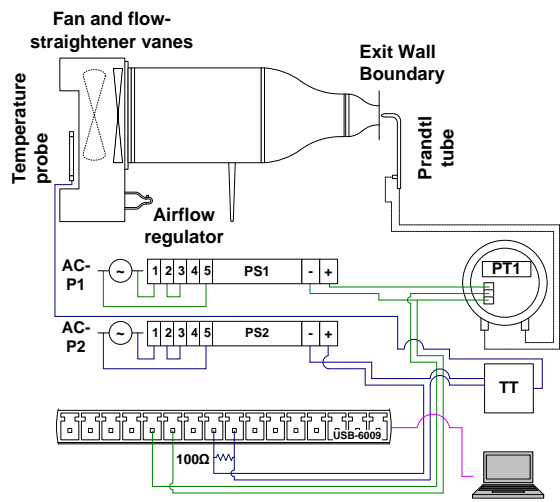

Fig. 1. The experimental setup.

Prandtl tube measurements were used to assess the mean axial velocity at the centerline of the jet and also the radial profiles of the axial velocity at small distances downstream of the jet exit. At these locations the axial velocity is dominant in the flow field and the levels of turbulence are very low, so that pressure variations in the radial directions are negligible. Before each experiment, the corresponding 'free-jet', i.e. the jet without the disk, was monitored. Measurements were carried out for two jet exit velocities, i.e. 14.5 and $19.5 \mathrm{~m} / \mathrm{s}$, resulting in different but of the same order of magnitude Reynolds numbers.

The total and static pressure ports of the Prandtl tube were connected to the high and the low pressure inputs of a Dwyer ${ }^{\circledR}$ differential pressure transmitter model MS-311-LCD. The transmitter is capable in measuring $1250 \mathrm{~Pa}$ maximum differential pressure with $1 \%$ accuracy. Time-series were monitored, collected and stored in a PC using an in-house developed LabView ${ }^{\circledR}$ virtual instrument connected to a National Instruments ${ }^{\circledR}$ multifunction data acquisition (DAQ) card. The experimental setup and the details of the measurement chain is shown in Fig. 1.

\subsection{Computational Modeling and Solution}

The computational domain designed to enclose the development of the jet flow along with the boundary conditions used in each face, are shown in Fig. 2. Outer boundaries were placed 110 and 55 jet diameters far from the exit while 15 jet diameters were used for the region upstream of the jet exit. A structured mesh accounting 74600 cells distributed properly with respect to the geometrical details of the jet facility was used for the domain. Axisymmetry was used to reduce the number of cells and consequently, the time spent for the simulations. Except of velocity inlet and pressure outlet conditions used for the jet exit and the right boundary of the domain respectively, all the external boundaries were set as pressure inlets. 


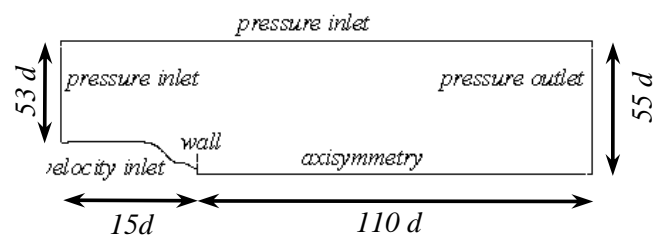

Fig. 2. Computational domain and boundary conditions.

Table 1 Simulations test cases

\begin{tabular}{ccccr}
\hline Re & $\begin{array}{c}\text { Exit } \\
\text { wall }\end{array}$ & $\begin{array}{c}\text { Turb. } \\
\text { model }\end{array}$ & Solver & $\begin{array}{c}\text { No. of } \\
\text { iterat. }\end{array}$ \\
\hline 50000 & $\times$ & $\mathrm{k}-\varepsilon$ & SIMPLEC & 2682 \\
& $\sqrt{ }$ & $\mathrm{k}-\varepsilon$ & SIMPLEC & 5612 \\
& $\times$ & $\mathrm{k}-\varepsilon$ & COUPLED & 412 \\
& $\sqrt{ }$ & $\mathrm{k}-\varepsilon$ & COUPLED & 1154 \\
& $\times$ & $\mathrm{R}-\mathrm{S}$ & COUPLED & 982 \\
& $\sqrt{ }$ & $\mathrm{R}-\mathrm{S}$ & COUPLED & 993 \\
\hline 65000 & $\times$ & $\mathrm{k}-\varepsilon$ & SIMPLEC & 2012 \\
& $\sqrt{ }$ & $\mathrm{k}-\varepsilon$ & SIMPLEC & 5003 \\
& $\times$ & $\mathrm{k}-\varepsilon$ & COUPED & 305 \\
& $\sqrt{ }$ & $\mathrm{k}-\varepsilon$ & COUPLED & 1012 \\
& $\times$ & $\mathrm{R}-\mathrm{S}$ & COUPLED & 749 \\
& $\sqrt{ }$ & $\mathrm{R}-\mathrm{S}$ & COUPLED & 626 \\
\hline
\end{tabular}

A number of tests were performed to assess the accuracy and the time-cost of different flow solvers and turbulence models (Table 1). Two solvers were utilized, i.e. Simplec (Patankar 1980) and Coupled (Lounsbury 2006). The latter was capable of reducing significantly both the number of iterations and the time spent for the convergence of the solution. Convergence criteria were always imposed to $10^{-4}$ for the mean and turbulent velocities and stresses respectively, while a $10^{-6}$ criterion was used for mass continuity. The residuals appeared to be sensitive with respect to the incorporated models and solvers. In particular when the Simplec algorithm was used, the residuals were characterized by oscillations for a large number of iterations before convergence, despite the proper manipulation of the corresponding relaxation factors. Two different turbulence models were incorporated, i.e. $\mathrm{k}-\varepsilon$ and Reynolds-Stress (RS) model, in order to predict except of the mean flow properties additional variables, such as the axial and radial normal stresses and, in particular, the <uv> turbulent shear stress. The total number of iterations for convergence and cpu time varied between 305 and 5612 or 4 and 42 minutes respectively using a $2.4 \mathrm{GHz}$, i5 processor with 4GB memory Dell® laptop pc. The test cases of the simulations carried out with Fluent ${ }^{\circledR}$ CFD software package and the efficiency of each computational scheme with respect to the number of iterations are shown in Table 1.

\section{Results}

All The decay of the mean streamwise velocity on the central axis of the jet is illustrated in Fig. 3. A logarithmic scale is used for the $y$-axis to demonstrate the linear variation of this variable with the axial distance just after a small region close to the exit that refers to the "potential core" of the jet. It is evident that the predictions of the k- $\varepsilon$ model combined with the coupled solver stand closer to the experimental measurements and present only minor differences considering the imposition of the exit wall boundary. The corresponding curves are almost identical showing a different trend compared to measurements, which seem to be systematic for the two test cases (i.e. jet with and without exit wall boundary) after the potential core of the jet for the higher Reynolds number. In contrast, Reynolds-stress model predicts a stronger influence of the exit wall boundary for both the Reynolds numbers, although it systematically over predicts the axial velocity decay compared to the experimental results. The latter indicate a slightly different behavior for the two Reynolds numbers. For the lower velocity, measurements of the jet with the exit wall boundary lie over those of the free jet, while the differences diminish with the axial distance, and for the higher Reynolds number minor differences are observed within the potential core, while the jet with the exit wall boundary seems to decay more rapidly in the far field

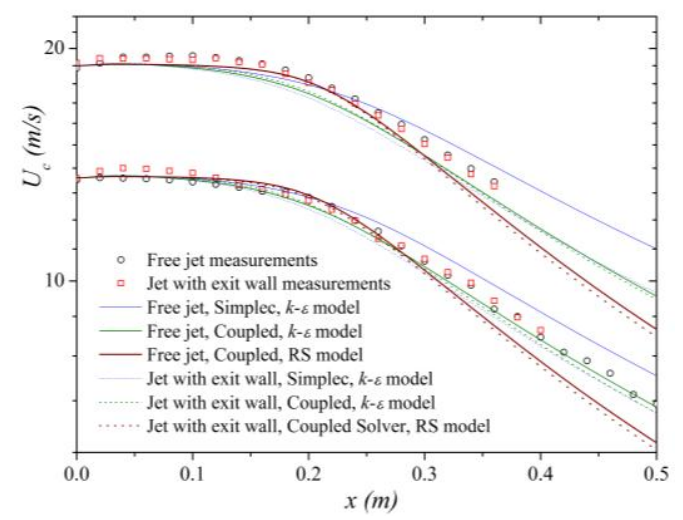

Fig. 3. Measurements and predictions of the axial velocity along the centerline of the jet.

Figure 4 presents isocontours of the axial velocity component, produced by interpolating Prandtl tube measurement data in the region of the initial development of the "free" jet that issues at $\mathrm{Re}=65000$ and those of the corresponding jet with the wall boundary imposed at exit. The same scale is used for the velocity range in both test cases. In this way, it is shown that only minor, insensitive divergences are present in the mean velocity distribution but also in the radial extent of the jet for a distance up to 7 diameters from jet exit.

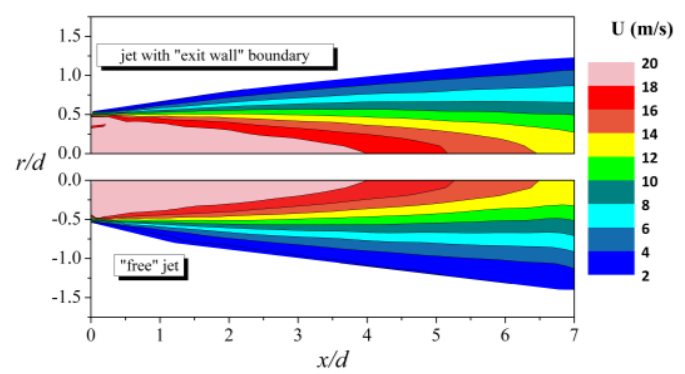

Fig. 4. Mean axial velocity contours in the near-field region. 


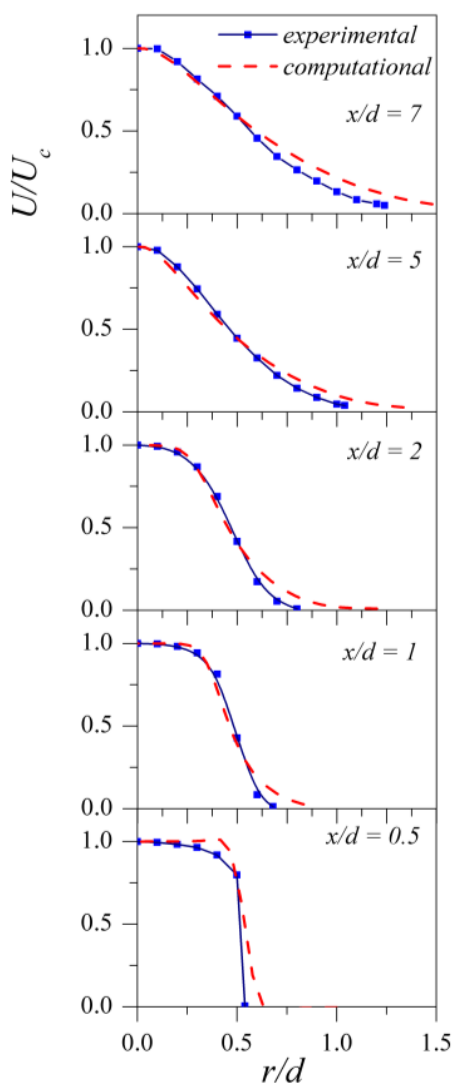

Fig. 5. Experimental and computational data of the mean axial velocity close to the jet exit.

The similar data of the mean velocity field provokes the use of advanced measuring techniques (capable of measuring turbulence) but also the implementation of computational fluid dynamics tools which have the capability to predict the turbulent properties of the two flows. Presently, we tried to gain insight into the flow structure of the two flows based on the "low cost" turbulence models described previously, which seem however to be capable of identifying systematic differences between the test cases considering in particular the turbulent flow properties for the different configurations. In addition, based on the trends observed in Fig. 3, in the following we will discuss systematically the influence of the exit wall boundary by utilizing the radial profiles of the mean and turbulent properties as they are predicted by the Reynolds stress model for the higher Reynolds number.

The agreement between the predictions of the Reynolds-Stress model and the measurements in the development region close to the exit for the jet flow that issues with $\mathrm{Re}=65000$ is presented in Fig. 5. Similar trends were observed in the profiles of the additional test cases, so that the latter are not presented here. Although it has been shown that this model over predicts the axial decay of the streamwise velocity, it is assumed as the most convenient to demonstrate the differences arising from the imposition of the exit wall boundary and furthermore, it gives the opportunity to discuss the turbulent flow properties, such as the normal and the shear, turbulent stresses.

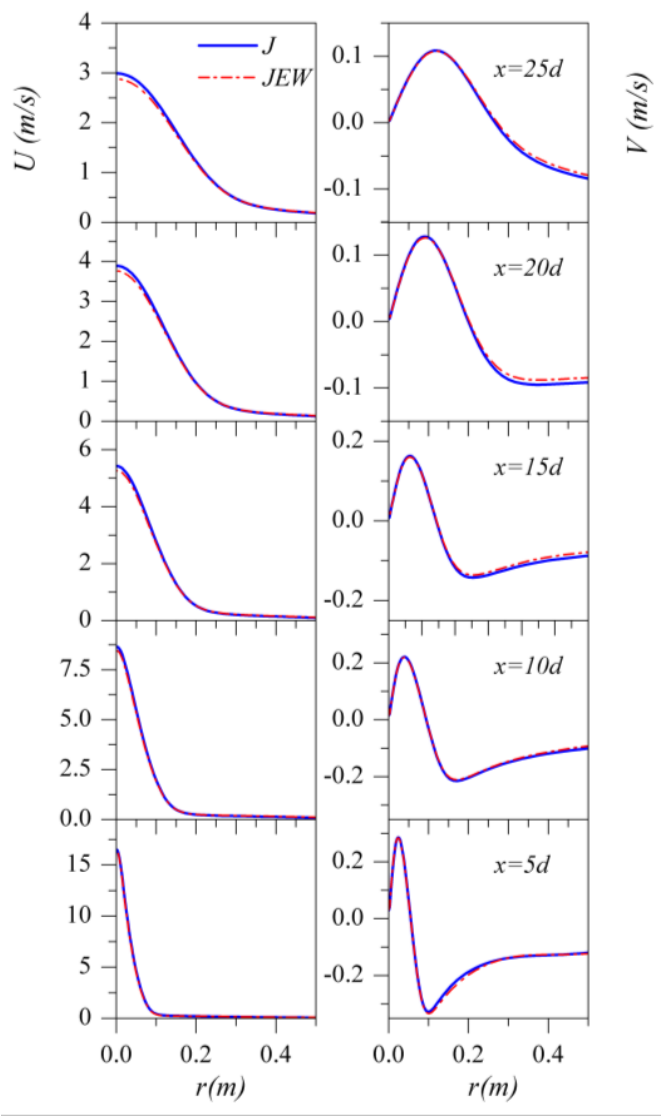

Fig. 6. Computed components of the mean velocity in discrete stations along the 'free' jet (J) compared to those when the exit wall boundary is present (JEW)

The predicted profiles of the streamwise and radial mean velocities in the region between 5 and 25 diameters from jet exit are presented in Fig. 6. No significant differences are observed for the two test cases, since the distributions seem to coincide until 10 diameters. After this distance, minor differences occur in the central part of the streamwise velocity and the outer part of the radial velocity component. In particular, when the exit wall boundary is imposed, the axial velocity is slightly lower close to the central axis of the jet (see also Fig. 3), while the radial velocity is insensitively higher in the outer part, indicating the slightly reduced entrainment of ambient surroundings from the jet flow.

More significant effects account for the turbulent flow properties when the exit wall boundary is imposed. Regarding the <uu> stress (Fig. 7), the distributions reach to systematically lower values in the central part of the jet. Similar trends characterize the radial normal stress $\langle\mathrm{vv}\rangle$, while for the shear stress <uv> the most significant differences occur in the vicinity of the offaxis peak, where also the free-jet flow presents a more effective mixing layer with the ambient surroundings. This behavior is expected, due to the zero value that corresponds to the central jet's axis for any jet flow considering this variable, and keeping in mind that the peak denotes the maximum shear of the velocity field. 

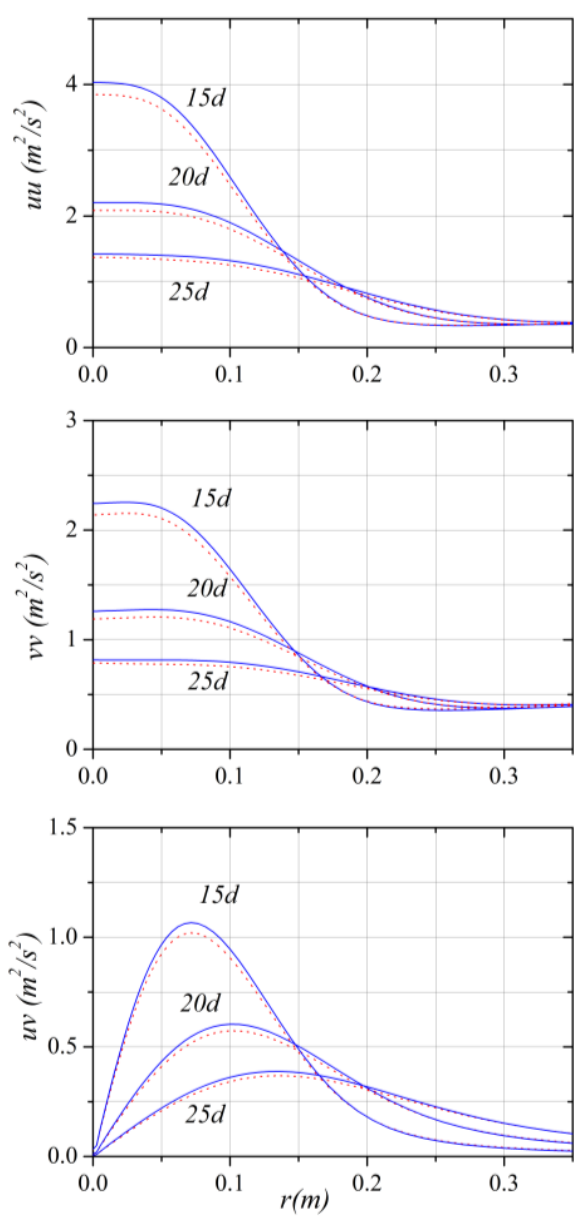

Fig. 7. Computational data of the jet with the exit wall boundary (red dot line) compared to the 'free' jet (blue line).

\section{Discussion}

The most important feature regarding the imposition of the exit wall boundary is shown in Fig. 8. The figure illustrates the predicted streamtraces in the vicinity of the jet exit. The circular disk is responsible for the formation of the recirculation zone behind the exit, which is expected to reduce the ability of the jet to entrain ambient fluid from the surroundings. The presence of this zone is expected to affect the initial development of the jet flow and also, the characteristics of the radial velocity profiles in the far field. However, by the present measurements and simulations it can be deduced that the addition of the wall at the exit has a rather minor influence on the mean velocity field while more systematic differences appear in the turbulent statistics.

The radial profiles of the mean and turbulent statistics are usually presented in a non dimensional form by utilizing the local velocity and length scales at each axial distance, i.e. the maximum or centerline velocity and the half-width of the jet, which is the radial distance at which the axial velocity has the half of the centerline value. In this way, it is possible to assess the selfsimilarity region, which is defined as the region where the non dimensional profiles of the flow properties coincide. In addition, by presenting the self-similar profiles of different experiments where the flow and geometry conditions vary, the influence of the initial conditions on the form of the self-similar characteristics and also, the distance at which the self-similarity is established is discussed. Although according to the classical theory of turbulence (Townsend, 1976), the effects of the initial conditions were expected to diminish in the far field, so that all the jets emanating with the same amount of momentum would reach a similar self-similarity state, during the last decades it has also been claimed that the initial conditions affect the development of the jet as well as its self-similarity state (George, 1989). These statements encouraged the present research, which except of the computational simulations presented here, include the experimental investigation of the influence of the exit wall boundary incorporating demonstrative, such as flow visualization, and advanced, i.e. hot-wire anemometry, techniques.

Figures 9 and 10 illustrate the non dimensional mean streamwise velocity and Reynolds shear stress 15, 20 and 25 diameters downstream from the jet exit, respectively. For the independent variable the axial distance from the jet exit is utilized instead of the half width of the jet, since after the potential core a linear variation between these two variables is established. Considering the mean velocity, the differences between the two test cases are reversed in comparison to the corresponding dimensional profiles, as the profile of the jet with the exit wall boundary stands systematically over that of the free-jet case. However, as the axial distance increase, the differences seem to diminish, while, in addition, the profiles for each test case tend to coincide, approaching a self-similar profile. Regarding the <uv> shear stress, the trend in the non dimensional profiles is similar, with the distributions of the free-jet case lying on lower values. The differences for the two test cases maintain moving downstream from the exit, indicating a stronger mixing layer for the jet with the exit wall boundary. Keeping in mind that this attribute refers to axial distances where the centerline streamwise velocity is equal for the two test cases, it can be explained by the tendency of the jet with the exit wall boundary to maintain its momentum although it entrains ambient fluid from a smaller region due to air "trapped" behind the wall boundary.

Reviewing the measurements of the most relevant of the literature (Abdel-Rahman et al. 1997), it has been shown that the jet with the wall boundary imposed at its exit delays to mix with the ambient fluid so that the decay of the mean axial velocity and the rate of radial spreading are lower compared to those of a free jet. As the mixing with the ambient is more vigorous for the free jet, the velocity decay along with the jet half-width results to a greater rate of mass entrainment. However, in the case of the jet with the exit wall boundary, it was also observed that at the edges of the jet the values of the mean axial velocity as well as those of the turbulent intensities were higher. This attribute could be possibly explained by the efforts of the "starving" from momentum (or mass) flow - as the jet with the exit wall boundary could be characterized - to entrain more actively ambient fluid peripherally since it cannot entrain fluid from the space behind its exit due to the imposed wall. 


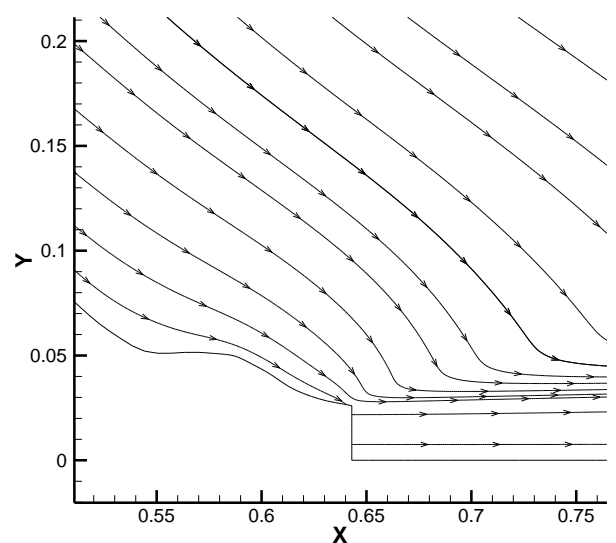

a) Free jet

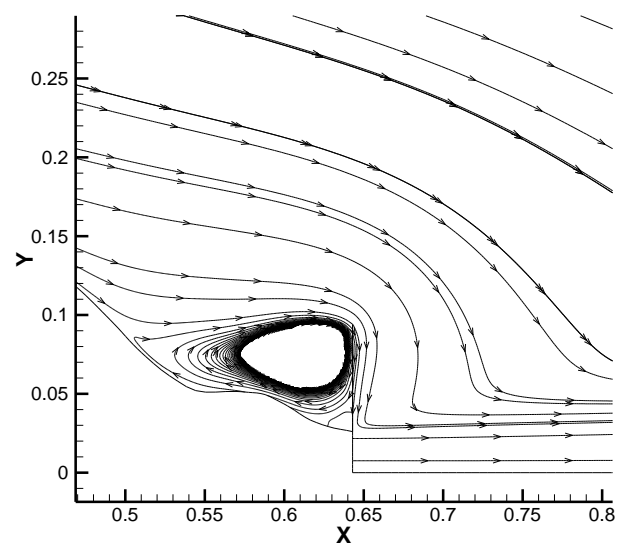

b) Jet with exit wall boundary

Fig. 8. Predicted streamtraces for the studied jet configurations.

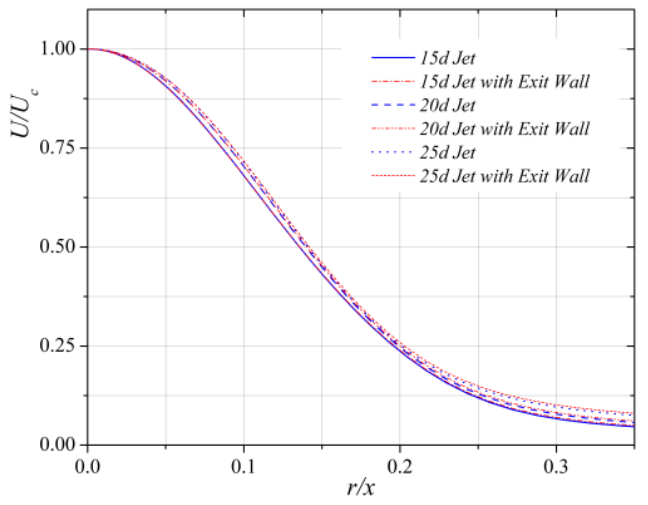

Fig. 9. Evolutions of the computed dimensionless mean axial velocities downstream of the contraction exit.

In the studies of Alnahhal et al. (2011) and Alnahhal and Panidis (2009) who used rectangular instead of axisymmetric jets, it has been shown that similar attributes can be observed but they depend on the aspect ratio of the jet and the Reynolds number. The utilization of the "endplates" results to a delay of mixing with the ambient for small aspect ratio $(A R=6)$ but the differences compared to the free jet do vanish when the Reynolds number increase. For higher aspect ratio $(A R=15)$, it was claimed that the results of the two flows (with and without endplates) are similar regarding the decay of the axial mean velocity and jet half-width, so that the entrained mass is also the same. The effect of the endplates can become significant only when additional "sidewalls" are imposed. Insignificant differences were also observed in the turbulent average statistics, with the values becoming slightly higher only when the endplates were used in combination with the sidewalls.

The present results partially validate the above described features. Regarding the mean axial velocity, the imposition of the wall boundary at the exit seems to have a minor effect, although it has the tendency to slightly decrease the values mainly at the centerline after the potential core (Figs. 3 and 6). This behavior is expected to affect the half-width of the jet which is obligated to move towards the edges of the jet (Fig. 6). On the other hand, although the mixing seems to be stronger with respect to the absolute values of the turbulent stresses at the same axial distances downstream of the jet exit (Fig. 7), the differences in the centerline mean axial velocity affect their nondimensional form, which is characterized by the trends described by the previous authors. In this form the turbulent stresses are higher denoting that for the same axial velocity, the jet with the exit wall boundary forms a stronger mixing layer with the ambient in order to recover the "loss" of fluid that stands behind the wall boundary and cannot be engulfed at the initial stages of its development.

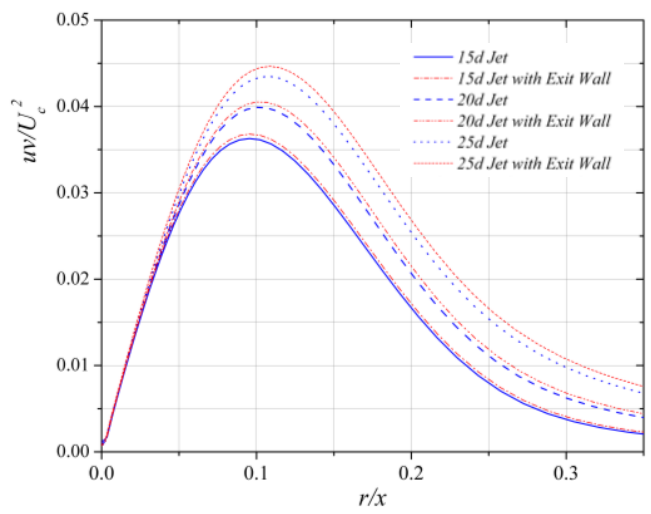

Fig. 10. Computed normalized Reynolds stresses component <uv> in the jet flow without and with the exit wall boundary.

\section{CONCLUSION}

Although jet's flow field constitutes a subject of research from the origins of the study of turbulence, recent findings indicate that the flow and geometry conditions can alter its development as well as the features of the self-similar profiles in the far field. The present study focuses on the effect of a wall boundary produced by a circular disk of twice the jet diameter imposed on the exit of the jet. Velocity distributions are analyzed and compared to those of the typical free jet, based on simulations utilizing different Fluent's ${ }^{\circledR}$ solvers. Supportive pressure measurements are used for the evaluation of the turbulence models. Results are presented in dimensional and non-dimensional form, in order to evaluate the effects of the exit wall boundary in 
absolute values and also with respect to the local velocity and length scales.

The predictions agree well with the experimental measurements at the centerline of the jet as well as in the region close to the exit. The results obtained by the different solvers and models indicate similar trends accounting for the imposition of the exit wall boundary on the original "free" jet flow. The Coupled solver seems to be more timely efficient, demonstrating high accuracy for both the turbulence models used in the simulations.

Computational results show that when the exit wall boundary is used, the mean streamwise centerline velocity decays slightly faster, while the radial profiles of the mean and turbulent velocity moments indicate that at the same distance from the orifice, the free-jet forms a stronger mixing layer with the surroundings. In contrast, when the non dimensional profiles are considered, the trends are reversed since the profiles for jet with the exit wall boundary lie on systematically higher values, indicating that for similar centerline streamwise velocities, it is this type of jet that interacts excessively with the ambient.

\section{REFERENCES}

Abdel-Rahman, A. (2010). LDA Measurements in the turbulent round jet. WSEAS Transactions of Fluid Mechanics, 5(4), 257-275.

Abdel-Rahman, A. A., W. Chakroun and S. F. AlFahed (1997). LDA measurements in the turbulent round jet. Mechanics Research Communications, 24(3), 277-288.

Alnahhal, M., A. Cavo, A. Romeos, K. Perrakis and Th. Panidis (2011). Experimental investigation of the effect of endplates and sidewalls on the near field development of a smooth contraction rectangular jet. European Journal of Mechanics B/Fluids, 30(4), 451-465.

Alnahhal, M. and Th. Panidis (2009). The effect of end plates on rectangular jets of different aspect ratios, Proceedings of the European Combustion Meeting, 1-6.

Antonia, R. A. and Q. Zhao (2001). Effect of initial conditions on a circular jet. Experiments in Fluids, 31(3), 319-323.

Borean, J. L., D. Huilier and H. Burnage (1998). On the effect of a co-flowing stream on the structure of an axisymmetric turbulent jet. Experimental Thermal and Fluid Science, 17(1-2), 10-17.

Borg, A., J. Bolinder and L. Fuchs (2001). Simultaneous velocity and concentration measurements in the near field of a turbulent lowpressure jet by digital particle image velocimetryplanar-laser-induced fluorescence. Experiments in Fluids, 31(2), 140-152.
Cowen E. A., K. A. Chang and Q. Liao (2001). A single-camera coupled PTV-LIF technique. Experiments in Fluids, 31(1), 63-73

George, W. K. (1989). The self preservation of turbulent flows and its relation to initial conditions and coherent structures, Hemisphere, Springer New York.

Kandakure, M. T., V. C. Patkar and A. W. Patwarhan (2008). Characteristics of turbulent confined jets. Chemical Engineering and Processing: Process Intensification, 47(8), 1234-1245.

Kwon, S. J. and I. W. Seo (2005). Reynolds number effects on the behavior of a non-buoyant round jet. Experiments in Fluids, 38(6), 801-812.

Lounsbury, R., J. (2006). Pressure based coupled solver in Fluent 6.3. In CFD Summit, Monterey, United States.

Panchapakesan, N. R. and J. L. Lumley (1993). Turbulence measurements in axisymmetric jets of air and Helium Part 1. Air Jet. Journal of Fluid Mechanics 246, 197-223.

Patankar, S. V. (1980). Numerical heat transfer and fluid flow. Hemisphere.

Quinn, W. R. (2006). Upstream nozzle shaping on near field flow in round turbulent free jets. European Journal of Mechanics B/ Fluids, 25(3), 279-301.

Todde, V., P. G. Spazzini and M. Sandberg (2009). Experimental analysis of low-Reynolds number free jets - Evolution along the jet centerline and Reynolds number effects. Experiments in Fluids, 47(2), 279-294.

Townsend, A. A. (1976). The Structure of Turbulent Shear Flow, Cambridge University Press.

Weisgraber, H. T. and D. Liepmann (1998). Turbulent Structure during transition to self-similarity in a round jet. Experiments in Fluids, 24(3), 210-224.

Wygnanski, I. and H. Fiedler (1969). Some measurements in the self preserving jet. Journal of Fluid Mechanics, 38(3), 577-612.

Xia, L. P. and K. M. Lam (2009). Velocity and Concentration measurements in initial region of submerged round jets in stagnant environment and in coflow. Journal of Hydro-Environmental Research, 3(1), 21-34.

Xu, G., R. A. Antonia (2002). Effect of different initial conditions on a round free jet. Experiments in Fluids, 33(5), 677-683. 